\title{
DESENVOLVIMENTO DE EMBALAGENS PARA MAQUIAGEM COM DESIGN BIOINSPIRADO E MATERIAIS BIODEGRADÁVEIS
}

[ Autor 1 ] Daniela Cristina Brioschi (daniela.brioschi@aluno.ufabc.edu.br) - Programa de Pós-Graduação em Engenharia e Inovação - UFABC

[ Autor 2 ] Rovilson Mafalda (rovilson.mafalda@ufabc.edu.br) - Programa de Pós-Graduação em Engenharia e Inovação - UFABC

[ Autor 3 ] Silvia Lenyra Meirelles Campos Titotto (silvia.titotto@ufabc.edu.br) - Programa de Pós-Graduação em Engenharia e Inovação - UFABC

\section{RESUMO}

O mercado de embalagens para cosméticos, sobretudo maquiagens, tem apresentado constante evolução em termos de design, uma vez que as embalagens representam importantes ferramenta de comunicação com o usuário. Nesse cenário, os plásticos tradicionais desempenham papel fundamental e possuem perspectiva de crescimento futuro, uma vez que são materiais leves, de custo relativamente baixo e com boas propriedades requeridas para diversas aplicações. Com base nesse contexto, faz-se necessária a utilização de novos materiais, que sejam capazes de minimizar os impactos ambientais causado pelos plásticos tradicionais, sendo os plásticos biodegradáveis apresentados como alternativas mais sustentáveis à utilização destes materiais. Para a pesquisa em andamento, estão sendo desenvolvidas, através do uso de metodologias apropriadas de design, sistemas CAD/CAE e elaboração de protótipos, embalagens para cosméticos com formas e funcionalidades inspiradas na natureza, com a incorporação de materiais biodegradáveis, obtendo um produto capaz de atender aos requisitos de design e sustentabilidade. Neste artigo são apresentados os resultados preliminares desta pesquisa e seus impactos esperados, nos quais as técnicas de prototipagem de produtos surgem como ferramentas potenciais no cenário de Internet das Coisas.

Palavras-chave: Design bioinspirado, materiais biodegradáveis, embalagens para cosméticos, internet das coisas

Área: $O$ design e o desenvolvimento de produtos e serviços

\section{INTRODUÇÃO}

As embalagens de produtos cosméticos e de cuidados pessoais tem experimentado evoluções ao longo do tempo e deixaram de ser apenas simples recipientes utilitários para se tornarem peças comerciais de arte que agregam valor ao produto e desempenham papel importante na tomada de decisão de compra pelo usuário (AUTTARAPONG, 2011; YABLONSKI \& MANCUSO, 2011).

De acordo com Auttarapong (2011), os fatores envolvidos nas decisões influenciadas pelas embalagens são: o conceito do produto, público-alvo, fator de identidade (design associado à uma determinada empresa ou grupo de empresas), elementos do design gráfico, fatores 
estéticos (processo de comunicação visual da embalagem do produto), valor agregado, fatores estruturais (de proteção/acomodação do produto), forma da embalagem, lucratividade e aspectos legais e aspectos ambientais de longo prazo.

Essas alterações de características desse mercado ao longo do tempo, no entanto, têm levado a um novo e dinâmico conceito de embalagem, também conhecidas como embalagens ecológicas ou embalagens "verdes" (YABLONSKI \& MANCUSO, 2011). Com o passar do tempo, os consumidores têm se tornado cada vez mais ambientalmente conscientes, de forma que as novas embalagens necessitam, além do apelo visual e sensual, ser ambientalmente amigáveis (YABLONSKI \& MANCUSO, 2011).

Como resultado desta evolução, alguns novos termos tornaram-se também chaves no universo de design como sustentabilidade, biodegradabilidade, reutilização e recarregamento (refis). Alguns especialistas definem as embalagens que utilizam o modelo de refil como uma das ideias mais promissoras em sustentabilidade em embalagens de cosméticos (YABLONSKI \& MANCUSO, 2011).

Atualmente, as principais preocupações com relação às embalagens ecológicas são (YABLONSKI \& MANCUSO, 2011):

- Preservação da compatibilidade e estabilidade;

- Preservação da permeabilidade e redução de peso;

- Tempo de prateleira e biodegradação;

- Integridade da embalagem;

- Contaminação pelo refil;

- Descarte adequado e infraestrutura para reciclagem;

- Impacto negativo em outros setores.

\subsection{Materiais biodegradáveis}

A crescente preocupação com a redução do número de rejeitos plásticos tem motivado o desenvolvimento de embalagens sustentáveis, definidas como embalagens "efetivamente recuperadas e utilizadas em ciclos biológicos e/ou industriais (KALE, 2011). Nesse contexto, os plásticos biodegradáveis aparecem como alternativas à utilização dos convencionais.

A literatura reporta as primeiras utilizações comerciais de polímeros biodegradáveis também nomeados por diferentes referências como biopolímeros - nos anos 1980. Os primeiros produtos foram produzidos a partir de polímeros convencionais, como o polietileno, aos quais foram incorporados amido ou outras substâncias de origem orgânica. Após o amido ser consumido pelos microrganismos, resultava em um produto final na forma de pequenos fragmentos (EBNESAJJAD, 2013; SMITH, 2005).

Os polímeros biodegradáveis têm sido avaliados ao longo dos últimos anos, no que se refere à sua definição. São definidos pela ASTM como "plásticos degradáveis nos quais a degradação resulta da ação de microrganismos de ocorrência natural, tais como bactérias, fungos e algas. Segundo a ISO, tal degradação resulta em fragmentos e baixa massa molar, ocasionado pela mesma classe de microrganismos referida. Entretanto, é válido ressaltar que os polímeros podem ser degradáveis sem que sejam biodegradáveis, isto é, podem desintegrar-se em pequenos fragmentos, sem que esse fenômeno seja resultante da ação de microrganismos (EBNESAJJAD, 2013).

A decomposição de polímeros biodegradáveis pode ocorrer por meio de rotas aeróbicas ou anaeróbicas, sendo que a degradação por compostagem ocorre de forma aeróbica, havendo 
consumo de oxigênio e matéria orgânica por microrganismos. Os polímeros compostáveis podem ser obtidos atráves de fontes renováveis, petróleo ou por uma mistura de ambos (KALE, 2011).

Com relação à utilização em embalagens, algumas empresas de manufatura e distribuição de cosméticos tem incorporados materiais biodegradáveis em suas embalagens. A Cargo Cosmetics, companhia de cosméticos com sede no Canadá, desenvolveu uma embalagem de batom baseada em poliácido láctico (PLA) - polímero produzido a partir de fontes renováveis, como o milho - com custos semelhantes ao das embalagens produzidas a partir do petróleo. Ao final do ciclo de vida, as embalagens podem ser descartadas em instalações de compostagem comercial (KALE, 2011).

\subsection{Design bioinspirado}

O design bioinspirado apresenta um promissor paradigma para inovação tanto em design quanto em design sustentável, utilizando analogias de sistemas biológicos para desenvolver soluções para problemas de engenharia. Tem ganhado importância como um movimento amplamente difundido no design, para um desenvolvimento consciente em termos ambientais (GOEL, MCADAMS \& STONE, 2014; GOEL, HELMS \& VATTAM, 2009, 2009).

Um dos principais aspectos do design bioinspirado é a utilização das similaridades entre a natureza e um problema específico, gerando não somente alguma inspiração e ideia de concepção para o designer, como também auxilia na escolha de modelo de solução dado um contexto com mais complexidade. A inspiração pelas formas da natureza pode ser de três diferentes formas: visual, conceitual e computacional. A inspiração visual, a mais utilizada e compreendida, é a que utiliza os aspectos visuais de um sistema biológico para a criação de sistemas de engenharia, com a mesma aparência. A inspiração visual é o uso de conhecimentos de sistemas biológicos para criar regras de design, padrões e princípios de funcionamento, exigindo entendimento tanto da natureza quanto da engenharia. A inspiração computacional, por sua vez, é a busca na natureza para encontrar algoritmos e representações (GOEL, MCADAMS \& STONE, 2014).

Do ponto de vista de design, existem alguns os fatores principais que tornam a abordagem bioinspirada como tema interessante de estudo e pesquisa (GOEL, HELMS \& VATTAM, 2009):

- Interdisciplinaridade do design bioinspirado, uma vez que este requer conhecimentos específicos em áreas de biologia e engenharia, e a capacidade de correlação entre estas áreas;

- Desafios de comunicação, uma vez que biólogos e engenheiros estudam diferentes áreas, com linguagens bastante próprias;

- Diferentes métodos de investigação e diferentes perspectivas sobre um mesmo problema, uma vez que, em geral, os biólogos possuem maior foco em compreender o design da natureza, enquanto engenheiros buscam novas formas de design que solucionem novos problemas;

- Design bioinspirado normalmente resulta em um design mais funcional e interdependente;

- Os recursos disponíveis na natureza, como materiais e processos, são diferentes dos disponíveis na área de engenharia. 
De forma prática, o design bioinspirado é responsável por uma série de designs úteis e inovadores, tendo como exemplo superfícies que repelem a água, inspiradas em uma folha de lótus com função autolimpante, e também o velcro, inspirada por uma espécie de planta (GOEL, MCADAMS \& STONE, 2014).

\subsection{Ferramentas computacionais}

No processo de desenvolvimento de produto, normalmente conduzido de forma sequencial, costuma-se encontrar a barreira denominada "paradoxo do design", que está relacionado à dicotomia entre o conhecimento do designer até aquele ponto do desenvolvimento e o número de decisões a serem tomadas. Por este motivo, normalmente são necessárias modificações no design do produto, conforme o design do produto evolui, sendo melhor compreendido (CHANG, 2014).

Nesse sentido, com o avanço das tecnologias computacionais, muitos testes físicos podem ser substituídos por simulações computacionais, reduzindo custos e diminuindo o tempo necessário para o desenvolvimento. Alguns softwares CAD/CAM/CAE (design, manufatura e engenharia assistidos por computador), como o SolidWorks ${ }^{\circledR}$, podem ser utilizados para auxiliar neste processo (CHANG, 2014).

$\mathrm{Na}$ indústria de embalagens cosméticas, ferramentas CAD/CAM também são utilizadas para o desenvolvimento de soluções. Um exemplo é a HK Cosmetic Packaging GmbH, que, além destas ferramentas, utiliza também metodologias de prototipagem (WEBPACKAGING, 2017).

\section{OBJETIVO}

O objetivo desta publicação é divulgar os resultados preliminares de uma pesquisa em andamento sobre aplicação de design bioinspirado para desenvolvimento de embalagens cosméticas, com foco em maquiagem.

\section{METODOLOGIA}

Para o desenvolvimento do trabalho, foi escolhida uma metodologia sistemática de design, consistindo de uma forma efetiva de racionalizar os processos de design e de produção e fornecendo soluções que possam ser replicadas, possibilitando também aumento da inventividade e a produtividade, uma vez que o sucesso é improvável sem a presença de elementos intuitivos do desenvolvedor (COUTO, 2010; BEITZ \& PAHL, 1996).

Um importante elemento na aplicação da metodologia sistemática é a estruturação do problema e das atividades, tornando mais fácil o reconhecimento de soluções já existentes e utilizadas anteriormente em projetos. $\mathrm{O}$ suporte computacional é também um elemento importante, uma vez que torna possível a divisão de atividades de forma efetiva e produtiva. (COUTO, 2010). As atividades em desenvolvimento ou a serem desenvolvidas envolvem as atividades como utilização de ferramentas computacionais, elaboração de protótipos, elaboração e aplicação de questionários e também a forma de análise dos resultados.

\subsection{Utilização de ferramentas computacionais}


A utilização de ferramentas computacionais tem como propósito a avaliação de dois importantes aspectos do produto desenvolvido: forma e funcionalidade. A forma inicialmente concebida a partir da bioinspiração está sendo desenvolvida através de modelagem bi e tridimensional, sendo adaptados os aspectos necessários. As etapas seguintes serão auxiliares na validação desta modelagem. A funcionalidade será avaliada por meio de simulações, de forma a verificar se o produto possui as características funcionais esperadas.

\subsection{Elaboração de protótipo}

Através da criação do protótipo deseja-se avaliar o quanto o produto final se mostra viável, quando comparado às expectativas iniciais - tanto em termos de forma, quanto de função. Além disso, será utilizado como forma de validar o conceito bioinspirado formulado e já modelado bi e tridimensionalmente na etapa anterior.

\subsection{Elaboração e aplicação de questionários}

A elaboração e aplicação de questionários, com posterior avaliação de resultados, tem como objetivo validar, com métricas definidas, o resultado do design concebido e desenvolvido sob a percepção de público externo aos autores do trabalho acadêmico.

\subsection{Forma de análise dos resultados}

Os resultados obtidos em cada uma das etapas serão analisados tendo sempre em vista os resultados da etapa anterior, conforme mencionado na seção 4 , acerca da metodologia. $\mathrm{O}$ esquema de análise seguirá o processo na Figura 1, a seguir:

Figura 1 - Fluxograma da forma de análise dos resultados. Fonte: Autor

Metodologia de design

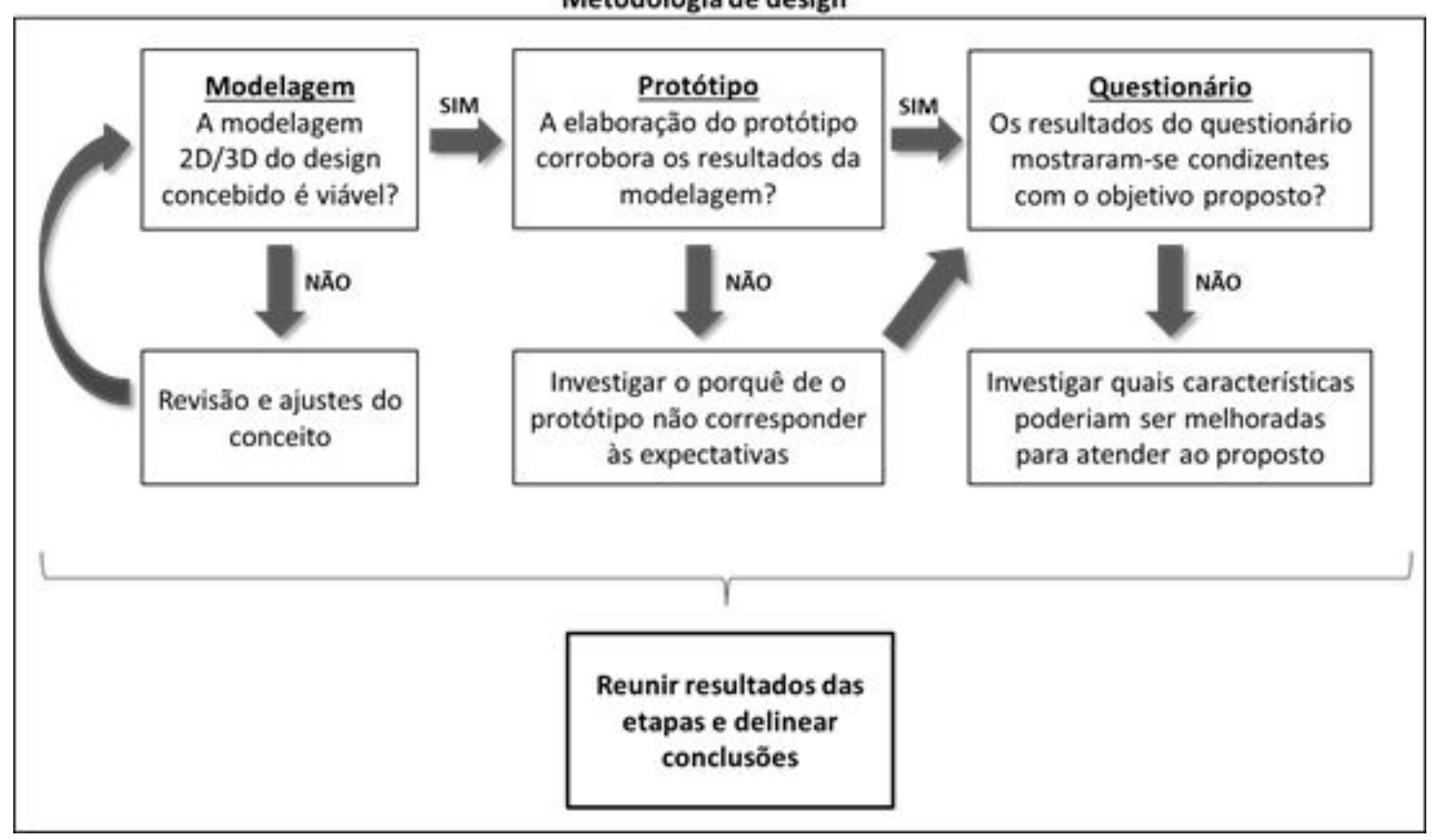




\section{RESULTADOS ESPERADOS}

Como resultado final, espera-se que o produto desenvolvido apresente características de forma e função relevantes, que sejam capazes de diferenciá-lo das embalagens cosméticas utilizadas até atualmente.

Em termos de metodologias de design, espera-se que a metodologia utilizada seja capaz de direcionar satisfatoriamente o processo de desenvolvimento, contribuindo para que este seja otimizado, promovendo oportunidades de melhorias e evitando retrabalhos, constituindo-se de uma ferramenta importante para a condução de desenvolvimento do produto.

Em termos de ferramentas $\mathrm{CAD} / \mathrm{CAE}$, espera-se que estas constituam ferramentas eficientes para a elaboração dos modelos e avaliação de propriedades funcionais, permitindo que o produto seja avaliado do ponto de vista de seus dois mais importantes aspectos: forma e função. É esperado que o produto final seja visualmente atraente, possuindo propriedades similares ou melhoradas em relação às embalagens atualmente utilizadas.

Com relação à elaboração de protótipo, espera-se que este possa corroborar o que foi projetado na etapa de modelagem $\mathrm{CAD} / \mathrm{CAE}$ em termos de design, uma vez que as propriedades do protótipo não serão avaliadas através de testes mecânicos ou térmicos. Os questionários também consistirão de uma forma de validação do design projetado.

\section{CONSIDERAÇÕES FINAIS}

Como trata-se de uma pesquisa em andamento com resultados de cunho qualitativo, que se propõe a realizar, no processo de design de uma embalagem de maquiagem, a integração de conhecimentos de áreas distintas, como design e materiais sustentáveis, já percebe-se na fase atual que integração de tais conhecimentos apresenta potencial de trazer ao universo de embalagens a possibilidade real de agregar ao design cada vez mais exigente em termos de forma e funcionalidade do mercado de maquiagens à materiais sustentáveis, capazes de reduzir significativamente o impacto ambiental do descarte de embalagens na natureza - sem comprometer, no entanto, o design do produto.

\section{REFERÊNCIAS}

AUTTARAPONG, D. Package Design Expert System Based on Relation between Packaging and Perception of Customer. Procedia Engineering, vol 3. 307-314. 2012.

BEITZ, W. PAHL, Gerhard. Engineering Design: a Systematic Approach. 2 ed. Springer-Verlag London. Inglaterra, 1996.

CHANG, Kuang-Hua. Design Theory and Methods using CAD/CAE: The Computer Aided Engineering. 1ed. Elsevier, 2015.

COUTO, Rita Maria de Souza. Modelo de Ensino de Métodos de Design de Produtos, Tese de Doutorado. Pontifícia Universidade Católica do Rio de Janeiro - PUC-Rio. Out. 2010.

EBNESAJJAD, S. Handbook Of Biopolymers And Biodegradable Plastics:Properties, Processing, And Applications. Waltham, Eua: Elsevier, 2013. 446 p. 
GOEL, Ashok K. HELMS, Michael. VATTAM, Swaroop S. Biologically inspired design: process. Design Studies, vol 30. 606-622p. Atlanta, 2009.

GOEL, Ashok K. MCADAMS, Daniel A. STONE, Robert B. Biologically Inspired Design: Computational Methods and Tools. Springer, Londres, 2014.

KALE, Gaurav. Chapter 21: Overview of Biodegradable Packaging, Methods and Current Trends. Formulating, Packaging and Marketing of Natural Cosmetic Products. John Wiley \& Sons. Hoboken, Eua, 2011.

MANCUSO, Sharon E. YABLONSKI, John I. Chapter 11: Microbial Risks and Eco-Friendly Packaging. Formulating, Packaging and Marketing of Natural Cosmetic Products. John Wiley \& Sons. Hoboken, Eua, 2011.

SMITH, Ray. Biodegradable polymers for industrial applications. Boca Raton, EUA: Woodhead Publishing Limited, 2005.

WEBPACKAGING - HK Cosmetic Packaging GmbH. Disponível em: $<$ https://www.webpackaging.com/en/portals/hkcosmeticpackaginggmbh/ > Acessado em 09 de abril de 2017. 